\title{
BMJ Open Associations between daily screen time and sleep in a racially and socioeconomically diverse sample of US infants: a prospective cohort study
}

\author{
Jennifer A Emond (D , , ${ }^{1,2}$ A James O'Malley, ${ }^{1,3}$ Brian Neelon, ${ }^{4}$ Richard M Kravitz, ${ }^{5}$ \\ Truls Ostbye, ${ }^{6}$ Sara E Benjamin-Neelon (1) ${ }^{7}$
}

To cite: Emond JA, O'Malley AJ, Neelon B, et al. Associations between daily screen time and sleep in a racially and socioeconomically diverse sample of US infants: a prospective cohort study. BMJ Open 2021;11:e044525. doi:10.1136/ bmjopen-2020-044525

- Prepublication history and additional supplemental material for this paper are available online. To view these files, please visit the journal online (http://dx.doi.org/10.1136/ bmjopen-2020-044525).

Received 05 September 2020 Accepted 02 June 2021

\section{Check for updates}

(c) Author(s) (or their employer(s)) 2021. Re-use permitted under CC BY-NC. No commercial re-use. See rights and permissions. Published by BMJ.

For numbered affiliations see end of article.

\section{Correspondence to}

Dr Jennifer A Emond;

Jennifer.A.Emond@Dartmouth. edu

\section{ABSTRACT}

Objective To determine the associations between screen media use and sleep throughout infancy (3-12 months).

Design Prospective Nurture birth cohort.

Setting North Carolina, USA, 2013-2015.

Participants Women enrolled in their second to third trimester, completed a phone interview after birth, and completed home visits at 3, 6, 9 and 12 months post partum.

Primary and secondary outcome measures Women reported the usual hours their infants slept during the day and night and their infants' usual use of five screen media activities at 3, 6, 9 and 12 months post partum. Adjusted mixed-effects regression analyses modelled the associations between infant screen time and sleep outcomes while disaggregating the between-infant and within-infant effects.

Results Among 558 mother-infant dyads, 374 (67.0\%) infants were black and 304 (54.5\%) households earned $<\$ 20000$ per year. Half (254, $50.2 \%$ ) of the infants engaged with screens at 3 months of age, while 326 (72.9\%) engaged at 12 months. The median value of the average daily screen time over the study period was 50 (IQR: 10-141) min. Infant screen time was inversely associated with nighttime sleep duration only when considering betweeninfant effects (adjusted beta: $-2.9 ; 95 \% \mathrm{Cl}-5.9$ to $0.0 ; p=0.054$ for log-transformed screen time). Effects were stronger for television+DVD viewing specifically (adjusted beta: $-5.2 ; 95 \% \mathrm{Cl}-9.1$ to $-1.4 ; \mathrm{p}<0.01$ for log-transformed television+DVD time). For example, an infant who averaged 1 hour of television+DVD viewing over the study period slept, on average, $9.20(95 \% \mathrm{Cl}$ 9.02 to 9.37 ) hours per night by 12 months compared with 9.60 (95\% Cl 9.41 to 9.80 ) hours per night for an infant with no screen time over the study period. There were no significant within-infant effects between screen time and night-time sleep, and screen time was not associated with daytime sleep or night-time awakenings.

Conclusions Screen time during infancy was inversely associated with night-time sleep duration; however, causal associations remain uncertain.

Trial registration number NCT01788644.
Strengths and limitations of this study

- This study reports on the associations between screen use and sleep among infants as they age from 3 to 12 months.

- Our analytic approach used repeated measures in the study to isolate the effects due to differences between infants versus differences within the same infant over time.

- Our analyses adjusted for a comprehensive set of potential confounding variables.

- Racial minorities and those from lower socioeconomic status were well represented in the cohort.

- The study relied on parent report and thus the precision of the exposure and outcome measures may be limited.

\section{INTRODUCTION}

Adequate sleep during infancy is essential for healthy cognitive, physical, emotional and behavioural development. ${ }^{1-3}$ Less night-time sleep has been associated with poorer cognitive development, ${ }^{12}$ socioemotional difficulties $^{3}$ and overweight/obesity risk ${ }^{4-6}$ in infants. Infant sleep patterns are highly dynamic in the first year of life. There is wide variability in sleep behaviours up to 3 months of age; thereafter regular circadian rhythms begin to develop. $^{7-9}$ Infants also shift to more nighttime and less daytime sleep and experience fewer night-time awakenings from 4 to 12 months. ${ }^{9-11}$ In a 2016 consensus statement, the American Academy of Sleep Medicine recommends that infants aged 4-12 months achieve $12-16$ hours of sleep per 24 hours. ${ }^{12}$ Identifying risk factors that may impede the natural development of sleep patterns during infancy is important to ensure optimal growth and development. 
There has been limited study of the influence of screen time on sleep during infancy ${ }^{13-18}$ despite a robust body of research demonstrating adverse effects of excessive screen time (eg, television (TV)/video viewing, playing apps/ games) on reduced sleep quantity and quality among children and adolescents. ${ }^{19}{ }^{20}$ In 2017 , children under the age of 2 years spent $42 \mathrm{~min}$ per day engaged with screens, with traditional TV as the primary source. ${ }^{21}$ In contrast, the American Academy of Pediatrics recommends no screen media for children under 18 months, ${ }^{22}$ largely due to potential adverse effects on cognitive, socioemotional and language development. ${ }^{23-26}$ Previous cross-sectional studies have reported inverse associations between screen time and night-time sleep ${ }^{13} 18$ in infants as young as 4 months. Inverse associations between infant screen time and 24-hour sleep have also been reported, ${ }^{13-18}$ with mixed findings for infants under 12 months; little research has examined screen use and daytime sleep (with mixed findings ${ }^{1318}$ or night-time awakenings. ${ }^{13}$

The goal of this study was to examine the associations between infant screen time and sleep quantity during the first year of life, leveraging the repeated-measures data from the prospective Nurture birth cohort study. We present an analytic approach that isolates the associations between screen time and sleep attributable to differences between infants and differences within the same infant over time. ${ }^{27}$ This approach allows us to address (1) if an infant's average screen time over the study year is associated with their average sleep over that same time frame; and (2) if an infant's observed screen time at one timepoint deviates from their expected screen time at that timepoint, their sleep at that timepoint is in turn affected. Using this analytic approach enables more refined statements regarding the associations by comparing an infant's usual screen time and sleep over the entire study year, as well as shorter-term deviations in those behaviours during a shorter time frame (ie, at each study assessment).

\section{METHODS}

\section{Participants}

The Nurture study ${ }^{28}$ is a longitudinal, observational birth cohort designed to examine feeding, activity and sleep in relation to excessive weight gain in infancy. Women were recruited from the county health department prenatal clinic and a private prenatal clinic in Durham, North Carolina, USA, during 2013-2015; data collection ended in 2017. Women were enrolled in their second or third trimester, completed a phone interview after birth, and completed home visits at 3, 6, 9 and 12 months post partum, at which time they also completed a questionnaire. A copy of the questionnaire is available from the corresponding author on reasonable request. Women provided written consent at enrolment and verbally confirmed consent for themselves and their infants at birth. The Nurture study was powered at 0.90 probability to detect a 0.09 increase in mean infant weight-for-length z-scores per each 10-hour-per-week increment spent in childcare outside the home, with 666 dyads enrolled and $85 \%$ retention over 12 months. ${ }^{28}$ The original protocol for the study is available in the online supplemental material and is published in BMJ Open. ${ }^{28}$

\section{Exposures}

Women reported their infants' screen time via questionnaire items at 3, 6, 9 and 12 months as the number of minutes their infants spent doing five screen activities on a typical weekday and typical weekend day, considering their infants' typical use over the past 4 weeks: (1) watching TV on a TV set; (2) watching DVDs or videotapes; (3) streaming videos or shows on TV, watching on a computer or a handheld device; (4) playing games on a handheld device; or (5) using educational software. Women were instructed to also consider their infants' screen media exposure at childcare when reporting each value. Weekday and weekend responses were weighted by $5 / 7$ and $2 / 7$, respectively, to compute a mean daily screen time per each category. Totals over all five categories were summed to create the total minutes of screen time per day. We included all screen time, including the use of educational software, to align with the American Academy of Pediatrics' recommendation of no screen time for children under the age of 18 months. ${ }^{22}$ Screen time was also categorised as TV+DVD viewing versus other (ie, streaming on a TV, computer or handheld device; playing games on a handheld device; using educational software) to best differentiate between screen time on a traditional TV set versus screen time on mobile devices. Infant screen time was not normally distributed and the distribution was positively skewed. There were several large outlying values and many parents reported no infant screen time. Therefore, screen time values were transformed for analysis. First, values $>10$ hours per day were truncated at 10 hours per day to align with the maximum allowable values reported in a previous study assessing media use and sleep among children. ${ }^{15}$ This process affected 55 $(2.9 \%)$ of all data points for infant screen time. Then, $1 \mathrm{~min}$ was added to all values to eliminate zeros and values were natural log-transformed.

\section{Outcomes}

Women reported the usual number of hours their infants slept during the day (from 07:00 to 19:00) and night (from 19:00 to 07:00) considering the past 4 weeks via questionnaire items at 3, 6, 9 and 12 months post partum. Durations were summed at each assessment to create total sleep per 24 hours. Women additionally reported the number of times their infants woke in the night (ie, night-time awakenings).

\section{Covariates}

Women reported their age, marital status, education and annual household income at enrolment and at each home visit. Infants' sex, race and ethnicity were reported at the postbirth phone call. Women reported on the following at each postpartum assessment, which were included as potential covariates: number of household members, infant breast feeding (yes/no), and weekly hours of formal and relativebased childcare. Women reported their own screen time at each assessment as the average number of hours they 
spent watching TV/DVDs/videotapes or streaming on a $\mathrm{TV}$, computer or handheld device, or playing games on a handheld device on a typical weekday and a typical weekend day; whether the mother's screen time was concurrent with the infant's screen time was not reported. Daily totals were similarly weighted and summed to create total screen time per day. The number of total media screens at home was also reported at 6 and 12 months post partum. Women reported their current cigarette smoking status (yes/no) and completed the Edinburgh Postnatal Depression Scale $(\text { EPDS })^{29}$ at each assessment. EPDS scores of 12 or more were considered a positive screen for postpartum depression. ${ }^{30}$ To assess overall disorder and environmental confusion at home, women completed the validated Confusion, Hubbub and Order Scale ${ }^{31}$ at 6 and 12 months post partum; higher scores indicate greater chaos. Potential confounders were selected to account for sociodemographic differences or because of potential associations with infant screen time or infant sleep. Specifically, exclusive breast feeding (vs partial or exclusive formula feeding) has been associated with more night-time awakenings at 3 and 6 months of age, ${ }^{32}$ more time spent in formal or informal childcare during the day has been inversely associated with night-time sleep duration among infants ${ }^{33}$ and delayed bedtime among infants and toddlers, ${ }^{34}$ maternal screen time is highly correlated with infant screen time ${ }^{35}$ maternal smoking has been related to night-time awakenings among infants, ${ }^{36}$ maternal depression has been related to unsettled sleep among infants, ${ }^{37}$ and a greater level of disorder and chaos at home has been related to sleep problems and fragmented sleep among young children. ${ }^{38}$ We included a category of 'missing' for each covariate to account for missing data. Covariates affected were maternal smoking status $(9.3 \%$ of dyads were missing data at all timepoints), maternal depression (10.3\% of dyads missing data at all timepoints) and maternal screen time (11.6\% of dyads missing data at all timepoints). We did not account for missing data for childcare measures because dyads missing data on those measures for all timepoints were minimal $(<0.5 \%)$. Our results from the adjusted regression models were similar when accounting for the missing data (as presented in the following section) and when the sample was limited to complete cases only (ie, without accounting for missing data), supporting that data on these covariates were missing completely at random. Additionally, values of 'missing' for these covariates were not related to the outcome in any model.

\section{Statistical analyses}

Analyses were completed on the subset of mother-infant dyads with both infant sleep and screen time reported for at least one postpartum assessment $(\mathrm{N}=558)$. We first completed a series of unadjusted analyses to identify potential covariates for the final models. We assessed the associations of infant, maternal and household characteristics with infant screen time (any vs none), and separately the associations of infant, maternal and household characteristics with each sleep outcome using unadjusted bivariate tests: $\chi^{2}$ test when comparing two categorical measures, t-test or one-way analysis of variance when comparing a continuous measure across a categorical measure, and Pearson's correlation coefficients when comparing two continuous measures.

Generalised mixed-effects regression was used to model the associations between infant screen time and each sleep outcome. Linear regression was used for nighttime, daytime and 24-hour sleep, and Poisson regression was used for the number of night-time awakenings. Infant screen time was included in each model in two ways. One, to compare screen time between infants (ie, between-infant effects), each infant's mean amount of screen time over the 1-year study period was included in the model. Second, to assess how differences in screen time within each infant over the study period related to sleep (ie, within-infant effects), we included a variable in the model that reflected each infant's deviation in screen time at each timepoint (ie, at 3, 6, 9 or 12 months of age) from their expected value of screen time at that timepoint. Specifically, the method of detrending ${ }^{27}$ was used, where a simple linear regression model was fit for each infant, regressing screen time on age (3, 6, 9 and 12 months), and residuals from that model reflected each infant's deviation in their expected value of screen time at each timepoint (ie, the difference between their observed screen time and their expected value based on their own linear regression). Including both of those measures of infant screen time in the model allowed us to isolate the between-infant and within-infant effects, respectively. ${ }^{27} \mathrm{~A}$ generalised form of the equation is included in the online supplemental material.

In each mixed-effects regression model, a random intercept and slope at age (ie, the time variable) were included for each infant. All models were adjusted for infant age and time-invariant sociodemographic characteristics measured at baseline (infant sex and race, mother's age at birth and educational level); annual household income was included as a time-varying covariate. A series of models further adjusted for three sets of time-varying covariates: infant breastfeeding status, maternal characteristics (smoking status, depression and daily screen time) and childcare measures (hours per day of relative or formal childcare, separately). The time-invariant covariates included were those measures associated with infant screen time or any sleep outcome at the $\mathrm{p}<0.10$ level in unadjusted analyses and were grouped into sets to determine how the main effect of infant screen time differed based on adjustment for different risk factors for poor sleep. A set of analyses were also completed to confirm that including infant screen time as logtransformed, versus non-transformed, improved the model fit as judged by Akaike's information criterion and inspecting the model assumptions (eg, homoscedasticity of residuals for linear regression models). A series of sensitivity analyses were also completed that confirmed there were no significant cross-lagged effects between infant screen media and sleep over time (eg, from 3 to 6 months of age), thus supporting our modelling approach 
to isolate the between-infant and within-infant effects. The adjusted models were also repeated among the dyads with complete data at each of the four postpartum study timepoints (ie, with infant sleep and screen time data reported at 3, 6, 9 and 12 months post partum). Analyses were completed using the $\mathrm{R}$ language for statistical computing (V.3.6.2).

\section{Patient and public involvement}

There was no patient or public involvement in the development of the research questions or in the analyses.

\section{RESULTS}

Among the 666 women who consented at birth with infants who met the inclusion criteria, 108 were excluded because they did not have any follow-up data on infant sleep or screen time for at least one postpartum visit, leaving 558 dyads in the analytic sample. Dyads included in the analysis were more likely to be white $(16.3 \%$ vs $9.3 \% ; \mathrm{p}=0.02$ ) and women were more likely to have some education past high school $(53.8 \%$ vs $43.9 \%$; $\mathrm{p}=0.08$ ) than dyads not included. The distribution of infant sex also differed between those included $(49.5 \%$ male $)$ versus excluded $(60.2 \%$ male) from the analysis $(\mathrm{p}=0.05)$. Included and excluded dyads were similar by other baseline measures at the $\mathrm{p} \geq 0.10$ threshold.

Table 1 includes the characteristics of the sample. There was an equal proportion of male and female infants in the sample, most $(67.0 \%)$ infants were black, the mean maternal age at enrolment was 27.5 years, about half $(53.8 \%)$ of women completed schooling after high school, and the annual household income was $\leq \$ 20000$ for $54.5 \%$ of dyads. Most $(452,81.0 \%)$ infants engaged with screens during at least one follow-up period (ie, at 3, 6, 9 or 12 months), with a median value for the average daily screen time over the entire postpartum study period of 50 (IQR: 10-141) min per day. Any screen time was more common among black infants and was associated with lower maternal age, non-marital or cohabitation status, lower maternal educational level, cigarette smoking, greater amount of maternal screen time, presence of postnatal depression among mothers at any study timepoint and lower annual household income (table 1).

The mean (SD) age of infants at each postpartum assessment was $3.4(0.5), 6.2(0.5), 9.4(0.5)$ and 12.3 (0.8) months, respectively. The proportion of infants with any screen time increased with age (table 2), from $50.2 \%$ of infants at 3 months to $72.9 \%$ at 12 months $(p<0.01)$.

Watching traditional TV or DVDs/videotapes accounted for most $(>70 \%)$ of infant screen time at any assessment. Streaming accounted for $15.5 \%$ and $15.7 \%$ of infant screen time at 3 and 12 months of age, respectively, and engagement with educational software accounted for $2.6 \%$ and $5.4 \%$ of screen time at 3 and 12 months of age, respectively. The median infant screen time over the 1-year study period was 50 (IQR: 10-141) min per day (also noted in table 1), with a mean of 109 min per day; the median infant TV+DVD time over the 1-year study period was $36 \mathrm{~min}$ per day, with a mean of $79 \mathrm{~min}$ per day. The disparities in the median and mean values reflect the positive skew of the data, yet we report the mean for comparison with other studies. When examining time-varying characteristics and infant screen time, the following were positively associated with any infant screen time over the study period: maternal smoking, depression and screen time and infant hours per week in relative-based childcare. Conversely, infant breast feeding and infant hours per week in formal childcare were inversely associated with any infant screen time. There were also significant temporal trends in all sleep outcomes (table 3). On average, infants increased their night-time sleep duration and decreased their daytime and 24-hour sleep duration with age. The number of infant night-time awakenings decreased with age.

There was a significant between-infant association between total infant screen time (min/day) and nighttime sleep duration in the adjusted linear mixed-effects regression models (table 4 ), where greater values of daily infant screen time over the study year were associated with shorter night-time sleep duration. The effect was stable across the varying adjustments for risk factors; however, the main effect was the most attenuated once adjusted for childcare characteristics relative to the model only adjusted for sociodemographic measures. Model coefficients require transformation for interpretation on the scale of the outcome. Figure 1 presents the predicted (ie, marginal) values from the adjusted regression model that included the childcare covariates (table 4, model 4) to facilitate interpretation of the effect size. For example, infants who averaged $15 \mathrm{~min}$ of daily screen time over the study period experienced $13.6 \mathrm{~min}$ less night-time sleep per night compared with infants with no screen time, and infants who averaged $60 \mathrm{~min}$ of daily screen time over the study period experienced $20.1 \mathrm{~min}$ less night-time sleep per night compared with infants with no screen time. An infant who averaged 1 hour of screen time per day over the study period would achieve, on average, 9.28 (95\% CI 9.13 to 9.42) hours of night-time sleep at 12 months of age, as compared with 9.61 (95\% CI 9.42 to 9.80 ) hours of night-time sleep for an infant with no screen time over the study period.

There were no significant within-infant effects in those models, meaning that each infant's deviation in screen time at any one timepoint, relative to their expected values of screen time at that timepoint, was unrelated to their night-time sleep. Infant race was also strongly associated with night-time sleep duration (table 4), where infants of black race averaged nearly 1 hour less nighttime sleep duration as compared with their white peers. Model findings were similar when limited to the 361 dyads with infant screen time and sleep reported at each postpartum timepoint (online supplemental table 1), in that there were significant between-infant effects, yet the within-infant effects were non-significant. For example, the adjusted association for the between-infant effect of 
Table 1 Infant, maternal and household characteristics overall and stratified by any infant screen time during the 12-month postpartum follow-up

\begin{tabular}{|c|c|c|c|c|}
\hline & \multicolumn{4}{|c|}{ Screen time at any timepoint during the 12-month follow-up } \\
\hline & \multirow{2}{*}{$\begin{array}{l}\text { Overall ( } \mathrm{N}=558) \\
\mathrm{n}(\%)\end{array}$} & \multirow{2}{*}{$\begin{array}{l}\text { None }(n=106) \\
n(\%)\end{array}$} & \multirow{2}{*}{$\begin{array}{l}\text { Any ( } n=452) \\
n(\%)\end{array}$} & \multirow[b]{2}{*}{$P$ value } \\
\hline & & & & \\
\hline $\begin{array}{l}\text { Average infant daily screen time (min) over the study } \\
\text { period, median (IQR) }\end{array}$ & $50(10-141)$ & 0 & $76(30-184)$ & - \\
\hline \multicolumn{5}{|l|}{ Infant characteristics } \\
\hline Male sex & $276(49.5)$ & $52(49.1)$ & $224(49.6)$ & $>0.99$ \\
\hline \multicolumn{5}{|l|}{ Infant race } \\
\hline White & $91(16.3)$ & $32(30.2)$ & $59(13.1)$ & \multirow[t]{4}{*}{$<0.001$} \\
\hline Black & $374(67.0)$ & $50(47.2)$ & $324(71.8)$ & \\
\hline Other & $92(16.5)$ & $24(22.6)$ & $68(15.1)$ & \\
\hline Missing/not reported & $1(0.2)$ & $0(0)$ & $1(0.2)$ & \\
\hline Hispanic/Latina ethnicity & $50(9.5)$ & $10(10.3)$ & $40(9.4)$ & 0.92 \\
\hline \multicolumn{5}{|l|}{ Maternal characteristics } \\
\hline Age at enrolment, years, mean (SD) & $27.5(5.8)$ & $29.6(6.2)$ & $27.1(5.6)$ & $<0.001$ \\
\hline \multicolumn{5}{|l|}{ Marital status at enrolment ${ }^{*}$} \\
\hline Married or cohabitating with partner & $337(60.7)$ & $79(75.2)$ & $258(57.3)$ & \multirow[t]{2}{*}{$<0.01$} \\
\hline Not married or cohabitating with partner & $218(39.3)$ & $26(24.8)$ & $192(42.7)$ & \\
\hline \multicolumn{5}{|l|}{ Education } \\
\hline High school graduate or lower & $258(46.2)$ & $32(30.2)$ & $226(50.0)$ & \multirow[t]{2}{*}{$<0.001$} \\
\hline Some college or higher & $300(53.8)$ & $74(69.8)$ & $226(50.0)$ & \\
\hline Cigarette smoker at any timepoint ${ }^{\star}$ & $162(29.0)$ & $19(17.9)$ & $143(31.6)$ & $<0.01$ \\
\hline $\begin{array}{l}\text { Daily screen time, average hours per day } \\
\text { over the study period }{ }^{\star} \text {, mean (SD) }\end{array}$ & $7.6(5.5)$ & $5.3(4.8)$ & $8.3(5.6)$ & $<0.001$ \\
\hline $\begin{array}{l}\text { Depression, EPDS } 12 \text { or more, at any } \\
\text { timepoint* }^{\star}\end{array}$ & $123(22.0)$ & $12(11.3)$ & $111(24.6)$ & $<0.01$ \\
\hline \multicolumn{5}{|l|}{ Household characteristics } \\
\hline \multicolumn{5}{|l|}{ Annual household income at enrolment* } \\
\hline$\leq \$ 20000$ & $304(54.5)$ & $39(36.8)$ & $265(58.6)$ & \multirow[t]{2}{*}{$<0.001$} \\
\hline$>\$ 20000$ & $208(37.3)$ & $63(59.4)$ & $145(32.1)$ & \\
\hline Missing & $46(8.2)$ & $4(3.8)$ & $42(9.3)$ & \\
\hline $\begin{array}{l}\text { Total household members, average over } \\
\text { the study period }{ }^{*}+\text { mean (SD) }\end{array}$ & $4.3(1.4)$ & $4.4(1.6)$ & $4.3(1.4)$ & 0.62 \\
\hline Number of screens at home†, mean (SD) & $7.9(3.3)$ & $7.9(3.1)$ & $7.8(3.3)$ & 0.82 \\
\hline Household $\operatorname{chaos}^{*} \dagger$, mean (SD) & $24.6(6.2)$ & $24.4(6.4)$ & $24.7(6.2)$ & 0.68 \\
\hline
\end{tabular}

Among 558 mother-infant dyads enrolled in the prospective Nurture birth cohort study.

Per cents sum down the columns to $100 \%$.

$P$ values are from $\chi^{2}$ test for categorical measures or t-test for continuous measures.

*Value treated as a time-varying covariate in later analyses.

†Average of values reported at 6 and 12 months post partum, which were the only timepoints when these measures were reported.

EPDS, Edinburgh Postnatal Depression Scale.

daily total screen time and night-time sleep was $-4.0(95 \%$ CI -7.9 to $-0.1 ; \mathrm{p}<0.05$ for log-transformed screen time) and the adjusted association for the within-infant effect of daily total screen time and night-time sleep was $1.1(95 \%$ CI -2.1 to $4.4 ; \mathrm{p}=0.50$ for log-transformed screen time); the model was adjusted for sociodemographic and childcare characteristics.
Table 5 presents the model results for the adjusted associations between infant screen time and nighttime, daytime and 24-hour sleep for all infant screen time combined and for infant screen time stratified as TV+DVD time and other screen time. The inverse main effect between infant screen time and night-time sleep was stronger when considering infant TV+DVD time 
Table 2 Unadjusted trends in infant screen time during the 12-month postpartum follow-up

Concurrent* screen time

\begin{tabular}{lllllc}
$\begin{array}{lllll}\text { Infant age } \\
\text { (months) }\end{array}$ & Overall $(\mathbf{n})$ & None, $\mathbf{n}(\%)$ & Any, $\mathbf{n}(\%)$ & viewing & Any screen time (min/day) \\
\hline 3 & 506 & $252(49.8)$ & $254(50.2)$ & 79.2 & Median (IQR) \\
\hline 6 & 476 & $195(41.0)$ & $281(59.0)$ & 72.6 & $1(0-120)$ \\
9 & 442 & $160(36.2)$ & $282(63.8)$ & 76.6 & $30(0-120)$ \\
12 & 447 & $121(27.1)$ & $326(72.9)$ & 73.3 & $60(0-149)$ \\
\hline P value for linear trend + & $<0.01$ & & 0.42 & $<0.01$ \\
\hline
\end{tabular}

Among 558 mother-infant dyads enrolled in the prospective Nurture birth cohort study.

${ }^{*}$ Concurrent screen time refers to screen time at that study assessment.

$\dagger P$ values for linear trends are from simple linear regression models regressing any screen time (\%), median screen time or proportion of screen time as television+DVD viewing on infant age (3, 6, 9 or 12 months).

specifically (adjusted beta: $-5.2 ; 95 \%$ CI -9.1 to -1.4 ; $\mathrm{p}<0.01$ for log-transformed TV+DVD time), independent of other screen time. Additionally, more TV+DVD screen time was related to less 24-hour sleep duration (adjusted beta: -6.4 ; 95\% CI -12.0 to -0.9 ; $\mathrm{p}=0.02$ for $\log$ transformed TV+DVD time). Other screen time was also positively associated with more 24-hour sleep duration (adjusted beta: $5.4 ; 95 \% \mathrm{CI}-0.6$ to 11.3 ; $\mathrm{p}=0.076$ for $\log$ transformed other screen time), although this finding was of borderline statistical significance. Model findings again were similar when limited to the 361 dyads with infant screen time and sleep reported at each of the four postpartum timepoints (online supplemental table 2), in that there were significant between-infant effects, yet the within-infant effects were non-significant. For example, the adjusted association for the between-infant effect of daily TV+DVD time and night-time sleep was -5.2 (95\% CI -10.1 to $-0.3 ; \mathrm{p}<0.05$ for log-transformed TV+DVD time) and the adjusted association for the within-infant effect of daily TV+DVD time and night-time sleep was $1.2(95 \%$ CI -2.5 to 4.9 ; $\mathrm{p}=0.52$ for log-transformed TV+DVD time); the model was adjusted for other infant screen time and sociodemographic and childcare characteristics.
However, in contrast to the model in table 5, the adjusted association for the between-infant effect of TV+DVD time and 24-hour sleep was not statistically significant in the model among the subsets with complete data (adjusted beta: $-5.5 ; 95 \%$ CI -12.4 to $1.5 ; \mathrm{p}=0.12$ for $\log$-transformed TV+DVD time).

Figure 2 presents the predicted, adjusted values of the sleep outcomes from those models to demonstrate the magnitude of the effect attributable to TV+DVD screen time; the predicted values are the marginal values from the final regression model for various values of infant screen time. Values for sleep outcomes are presented for 12 months of age. Infants who averaged $60 \mathrm{~min}$ of daily TV+DVD time over the study period experienced $24.4 \mathrm{~min}$ less night-time sleep per night compared with infants with no TV+DVD screen time over the study, adjusted for screen time other than TV+DVD time (figure 2A). There were no significant associations between infant screen time and daytime sleep duration (figure 2B). Infants who averaged $60 \mathrm{~min}$ of daily TV+DVD time over the study period experienced $29.1 \mathrm{~min}$ less 24-hour sleep compared with infants with no TV+DVD screen time over the study, adjusted for screen time other than TV+DVD time

\begin{tabular}{|c|c|c|c|c|c|}
\hline \multirow{2}{*}{$\begin{array}{l}\text { Infant age } \\
\text { (months) }\end{array}$} & \multirow[b]{2}{*}{ Overall (n) } & \multirow{2}{*}{$\begin{array}{l}\begin{array}{l}\text { Night-time sleep } \\
\text { (hours)* }\end{array} \\
\text { Mean (SD) }\end{array}$} & \multirow{2}{*}{$\begin{array}{l}\text { Daytime sleep } \\
\text { (hours) } \dagger \\
\text { Mean (SD) } \\
\end{array}$} & \multirow{2}{*}{$\begin{array}{l}\begin{array}{l}\text { 24-hour sleep } \\
\text { (hours/day) }\end{array} \\
\text { Mean (SD) }\end{array}$} & \multirow{2}{*}{$\begin{array}{l}\text { Number of night-time } \\
\text { awakenings } \\
\text { Mean (SD) }\end{array}$} \\
\hline & & & & & \\
\hline 3 & 506 & $8.6(1.7)$ & $5.1(2.2)$ & $13.8(2.7)$ & $2.0(1.3)$ \\
\hline 6 & 476 & $9.0(1.6)$ & $4.0(1.9)$ & $13.0(2.3)$ & $1.7(1.4)$ \\
\hline 9 & 442 & $9.2(1.5)$ & $3.6(1.6)$ & $12.8(2.2)$ & $1.6(1.3)$ \\
\hline 12 & 447 & $9.3(1.4)$ & $3.3(1.5)$ & $12.6(1.8)$ & $1.4(1.1)$ \\
\hline$P$ value for & $d \neq$ & $<0.05$ & $<0.05$ & 0.07 & $<0.05$ \\
\hline
\end{tabular}

Among 558 mother-infant dyads enrolled in the prospective Nurture birth cohort study.

${ }^{*}$ Night-time sleep reflects sleep from 19:00 to 07:00.

†Daytime sleep reflects sleep from 07:00 to 19:00.

$\ddagger P$ values for linear trends are from simple linear regression models regressing night-time sleep, daytime sleep, 24-hour sleep, achieving 12 or more hours of sleep per night (\%) or number of night-time awakenings on infant age (3, 6, 9 or 12 months). 
Table 4 Adjusted longitudinal associations of total infant screen time and night-time sleep while disaggregating the betweeninfant and within-infant effects

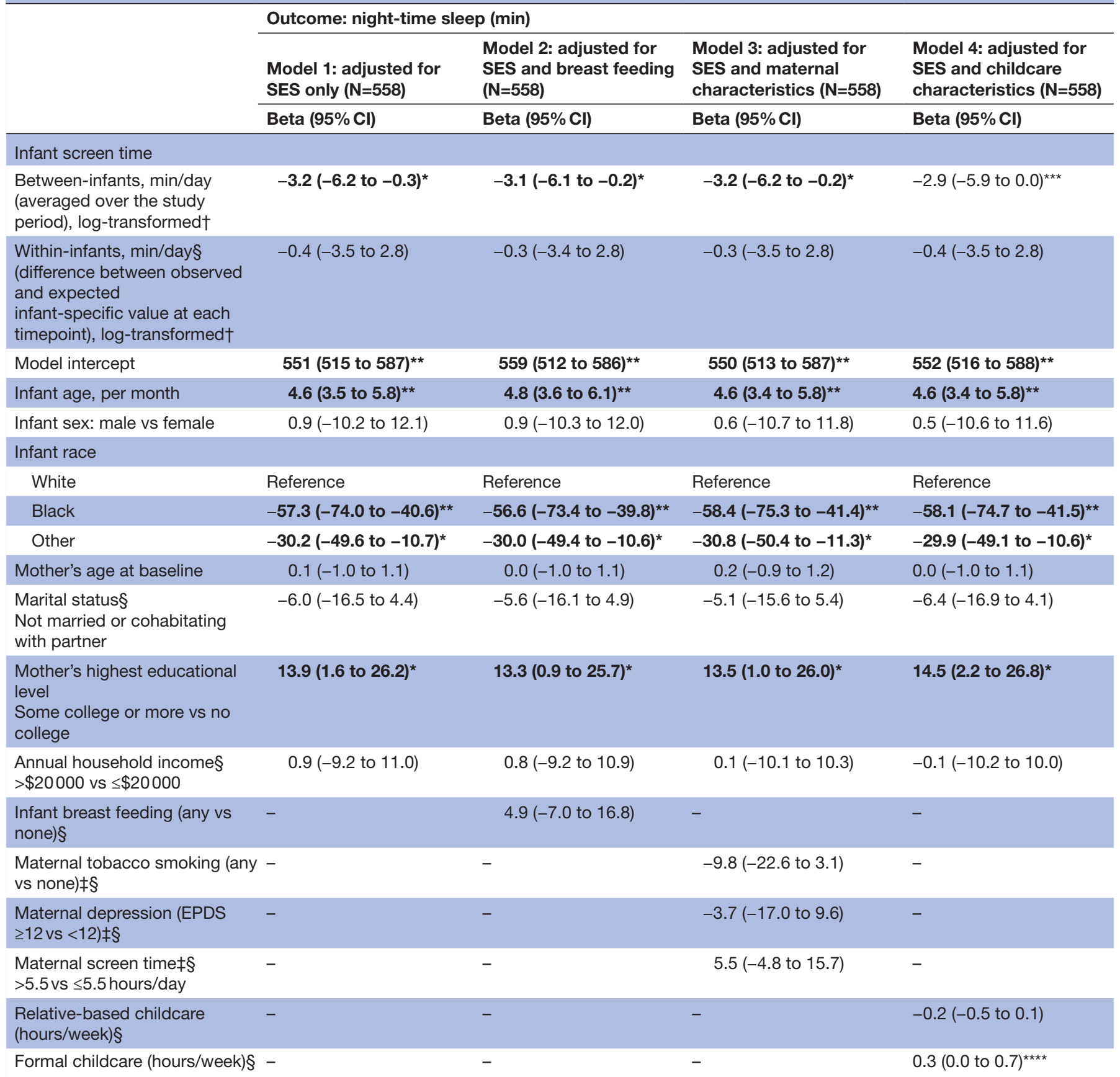

Each column reflects one mixed-effects linear regression model with a random effect at the infant level and a random slope for age (ie, time). All model covariates are presented.

${ }^{*} \mathrm{P}<0.05,{ }^{* *} \mathrm{P}<0.01,{ }^{* * *} \mathrm{P}=0.054,{ }^{* * *} \mathrm{P}=0.08$.

†The predictors are on the natural log scale and are interpreted as the change in night-time sleep per $1 \%$ increase in infant screen time. For example, a $1 \%$ incremental difference in infant screen time between infants translates into a difference of approximately -0.03 min of night-time sleep. Figure 1 presents the effect size in absolute terms.

$\ddagger$ A category of 'missing' was included for these categorical variables (breastfeeding data were not missing in this analytic subset). Effect sizes for those 'missing' categories are not included in the table for simplicity; however, none of those 'missing' categories was significantly associated with the outcome at the $p<0.05$ level. A category of 'missing' was not included for hours of relative-based or formal childcare, for which missing data were minimal $(<0.5 \%)$. There was one infant missing race and that infant was classified as 'other' race for the regression models.

$\S$ Time-varying covariate.

EPDS, Edinburgh Postnatal Depression Scale; SES, socioeconomic status. 


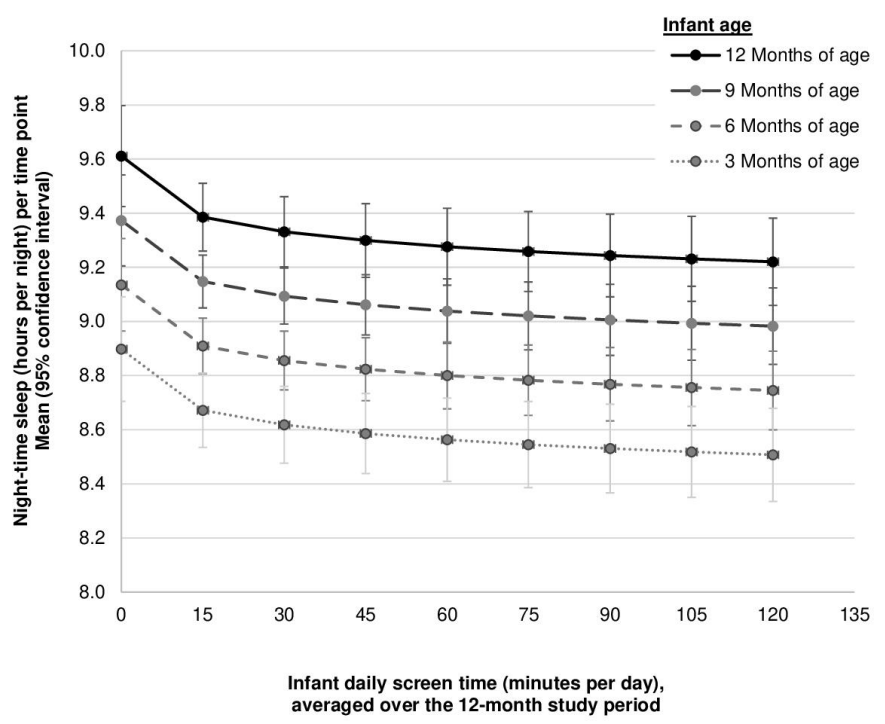

Figure 1 Predicted, adjusted values of night-time sleep, stratified by infant age, for a range of mean daily screen time (all screen time combined) over the study period; infants were enrolled in a prospective cohort study. Predicted values are the marginal values from a mixed-effects linear regression model adjusted for sociodemographic characteristics (infant age, sex and race; maternal age, marital/cohabitation status and education; and annual household income) and childcare characteristics (hours/week in relative-based or formal childcare, separately). Marital/cohabitation status, annual household income and childcare characteristics were timevarying covariates. Random effects at the infant level and a random slope for age were included in the model. Infant screen time was included as log-transformed and included as two terms to account for the between-infant and withininfant effects. The predicted values in this figure reflect the between-infant differences in night-time sleep for various values of screen time ( $\mathrm{min} /$ day) among infants, with screen time reflecting the mean daily screen time per day over the study period (ie, the between-infant differences; see table 4, model 4). Data are from 558 dyads with data collected during at least one postpartum follow-up; not all dyads have complete data at each timepoint. The following are the number of infants for each range of average daily screen time over the study period: $0 \mathrm{~min} /$ day: $n=106 ; 1-15 \mathrm{~min} /$ day: $n=66 ; 16-30 \mathrm{~min} /$ day: $n=52 ; 31-45 \mathrm{~min} /$ day: $n=42 ; 46-60 \mathrm{~min} /$ day: $n=39 ; 61-75 \mathrm{~min} /$ day: $n=25 ; 76-90 \mathrm{~min} /$ day: $n=30$; 91-105 min/day: $n=22 ; 106-120 \mathrm{~min} /$ day: $n=19 ; 120+\mathrm{min} /$ day: $n=157$.

(figure 2C). Finally, there were no associations between infant screen time and the number of night-time awakenings when considering all screen time combined or stratified by TV+DVD and other screen time (data not shown).

\section{DISCUSSION}

In this diverse sample of 558 mother-infant dyads, most infants engaged with screens from 3 to 12 months of age, and infant screen time was associated with less nighttime sleep in the first year of life. Infants who averaged more daily screen time over the study year experienced less night-time sleep than their peers over that same time frame, and the effect was greatest when considering TV+DVD viewing. Specifically, infants who averaged 60 min of daily TV+DVD time over the study period experienced approximately $24 \mathrm{~min}$ less night-time and $29 \mathrm{~min}$ less 24-hour sleep compared with infants with no TV+DVD screen time during infancy (ie, 3-12 months of age). Effects were consistent when considering adjustment for multiple covariates. Importantly, we did not find evidence of within-infant effects, meaning that deviations in screen time at any one specific timepoint (ie, 3, 6, 9 or 12 months of age), as compared with an infant's average screen time over the study period, were not uniquely related to sleep. Our findings align with a previous study among older children ${ }^{15}$ that demonstrated a significant association between greater TV viewing and shorter sleep duration as children aged from 1 to 7 years, where that effect was largely attributable to between-child differences that represented children's average TV viewing over the study period. In summary, our findings document that screen time increases considerably during infancy and suggest that more screen time, on average, in infancy relates to less night-time sleep, on average. Effects were limited to between-infant differences. In contrast, shorter-term deviations in screen use did not have a more acute effect on sleep, independent of an infant's usual screen time over the study year.

Our reported inverse association between screen time and night-time sleep duration aligns with the few previous studies among infants ${ }^{131718}$ and the multitude of studies among preschool-aged and older children. ${ }^{19}$ In a crosssectional study of 4-month-old infants, ${ }^{18}$ each hour of screen use was related to $13 \mathrm{~min}$ less night-time sleep duration. In a cross-sectional study of 715 parents of children aged 6-36 months old, ${ }^{13}$ each additional hour of infant touchscreen tablet use related to $26.4 \mathrm{~min}$ less night-time sleep duration; that study did not include the child's TV viewing but did adjust for background TV exposure. It is difficult to directly compare our findings with those previous studies because of the way infant screen time was treated: in the two previous studies, ${ }^{13} 18$ infant screen time was treated as a continuous, normally distributed variable, while in our study infant screen time was zeroinflated and highly skewed and was therefore natural logtransformed. Yet effect sizes across the studies are similar. We did not find evidence that infant screen time related to daytime sleep duration. That null association aligns with the previous study of 4 -month-olds, ${ }^{18}$ yet differs from the survey of children aged 6-36 months old, ${ }^{13}$ in which each additional hour of infant screen time related to 10.8 more minutes of daytime sleep. We also report that infant screen time was unrelated to night-time awakenings, a finding similar to that same previous study of children aged 6-36 months old. ${ }^{13}$ In summary, evidence appears to align that greater levels of infant screen time are associated with shorter night-time sleep duration, yet associations with other sleep outcomes are less consistent. However, additional studies specifically assessing between-infant and within-infant effects are needed to 
Table 5 Adjusted longitudinal associations between infant screen time and night-time, daytime and 24-hour sleep duration among infants, while disaggregating the between-infant and within-infant effects, with screen time modelled as (A) any screen time combined or (B) stratified as TV+DVD or other screen time

\begin{tabular}{|c|c|c|}
\hline $\begin{array}{l}\text { Outcome: night-time } \\
\text { sleep (min) }(\mathbf{N}=558)\end{array}$ & $\begin{array}{l}\text { Outcome: daytime sleep } \\
\text { (min) }(\mathbf{N}=558)\end{array}$ & $\begin{array}{l}\text { Outcome: } 24-\text { hour } \\
\text { sleep (min) }(\mathbf{N}=558)\end{array}$ \\
\hline $\begin{array}{l}\text { Mean difference } \\
\text { Beta }(95 \% \mathrm{Cl})\end{array}$ & $\begin{array}{l}\text { Mean difference } \\
\text { Beta }(95 \% \mathrm{Cl})\end{array}$ & $\begin{array}{l}\text { Mean difference } \\
\text { Beta }(95 \% \mathrm{Cl})\end{array}$ \\
\hline
\end{tabular}

(A) Modelled as all screen time combined

All screen time combined

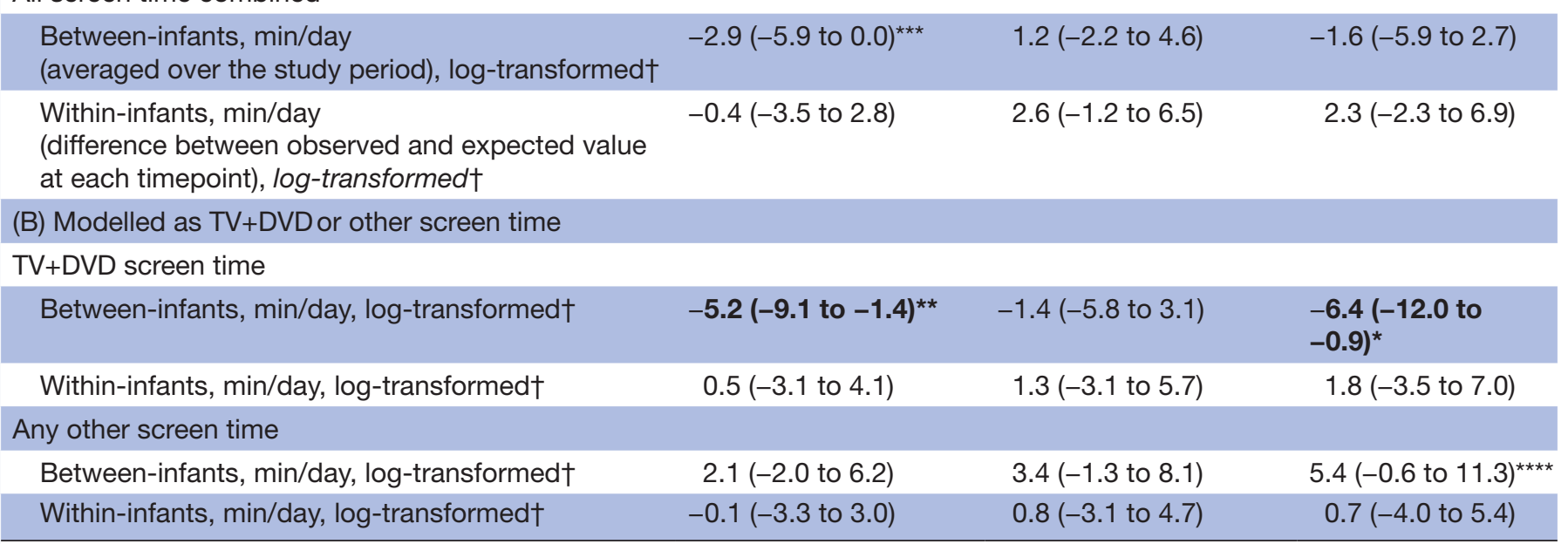

Two models (A and B) for each outcome are presented. Each model is a mixed-effects linear regression model with random effects at the infant level and a random slope for age (ie, time). Each model was adjusted for sociodemographic (infant age, sex and race; maternal age, marital/cohabitation status and education; and annual household income) and childcare characteristics (hours/week in relative-based or formal childcare, separately). Marital/cohabitation status, annual household income and childcare characteristics were time-varying covariates. Other screen time includes streaming videos or shows on a television (TV), computer or handheld device; playing games on a handheld device; or using educational software.

${ }^{*} \mathrm{P}<0.05,{ }^{* *} \mathrm{P}<0.01,{ }^{* * *} \mathrm{P}=0.054,{ }^{* * * *} \mathrm{P}=0.076$.

†The predictors are on the natural log scale and are interpreted as the change in nighttime sleep per a $1 \%$ increase in infant screen time.

better understand if our findings are generalisable across different populations.

TV+DVD viewing accounted for most of infants' screen time in our study, and we found that infant TV+DVD viewing had a greater association with shorter nighttime sleep duration than all screen time combined. Our measure of screen time other than TV+DVD viewing approximated mobile or handheld media, media that may have been more challenging for parents to report accurately as compared with TV+DVD viewing, and thus may have been more affected by reporting bias. Mobile media use among infants also requires parental (or other caregiver) involvement and may involve more active interaction from the infant. Thus, mobile screen use may be qualitatively different from when infants more passively view TV.

Our findings suggest that children with higher levels of screen time, on average, over the study period had lower amounts of night-time sleep, on average. Our study cannot prove a causal relationship exists between screen time and sleep. It is possible that reverse causality explains the reported association, where less night-time sleep led to more screen time. It is also possible that unmeasured confounding is related to both increased infant screen time and decreased night-time sleep, which would explain our observed association for the between-infant effect. However, our finding of a significant between-infant effect was consistent after adjusting for a multitude of other risk factors, suggesting unmeasured confounding may be low. The lack of a significant within-infant effect, where deviations in screen time at any one specific timepoint (ie, 3, 6, 9 or 12 months of age), as compared with an infant's average screen time over the study period, were not uniquely related to sleep, further suggests that more acute and thus potentially causal associations are not present between screen time and sleep. However, our measurements of infant screen time and sleep were both parent-reported and may lack the precision needed to measure small to moderate changes in screen time or sleep over time. Thus, it is possible that measurement error obscured any within-infant effects over the study period. Importantly, negative effects of screen time on infant sleep are biologically plausible. ${ }^{19}$ Screen time at night or in the bedroom may displace sleep, exposure to stimulating media content may disrupt sleep, ${ }^{39} 40$ and exposure to light emitted from screens may disrupt sleep hormones and circadian cycles. Unfortunately, we did not record when infants used screens, and we are not able to 

A) Outcome: Night-time sleep at 12
months of age
B) Outcome: Daytime sleep at 12 months of age
C) Outcome: 24-Hour sleep at 12 months of age

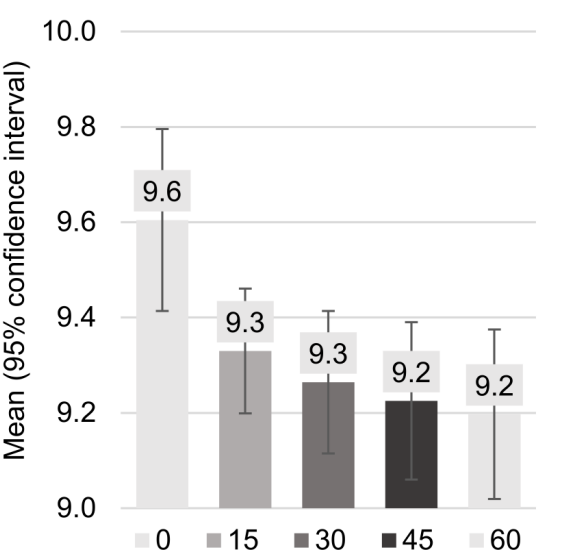

Average daily infant TV+DVD time (minutes per day)

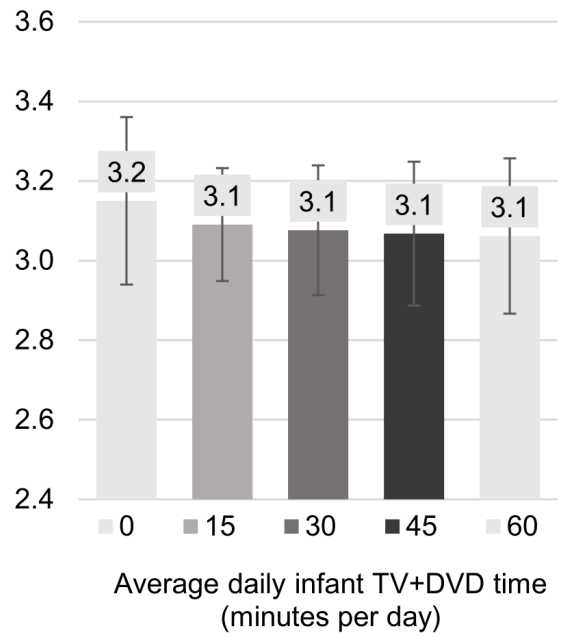

13.4

13.2

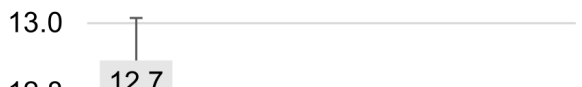

12.8

12.6

12.4

12.2

12.0

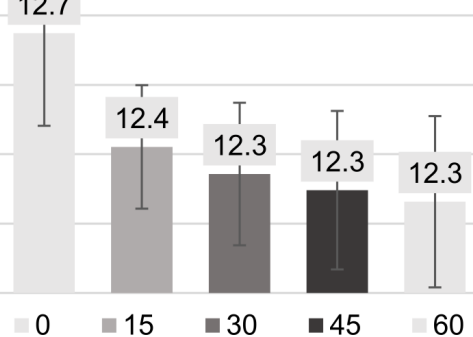

Average daily infant TV+DVD time (minutes per day)

Figure 2 Predicted, adjusted mean values of (A) night-time, (B) daytime and (C) 24-hour sleep at 12 months of age, by average daily television (TV)+DVD screen time over the study period. Values are adjusted for other infant screen time. Predicted values are the marginal values from separate mixed-effects linear regression model $(n=555)$ adjusted for sociodemographic characteristics (infant age, sex and race; maternal age, marital/cohabitation status and education; and annual household income) and childcare characteristics (hours/week in relative-based or formal childcare, separately). Marital/cohabitation status, annual household income and childcare characteristics were time-varying covariates. Random effects at the infant level and a random slope for age were included in the model. TV+DVD time was included as a log-transformed variable in the regression model and was included as two terms to account for the between-infant and within-infant effects; each model was also adjusted for all other (ie, non-TV+DVD) screen time. The predicted values in this figure reflect the mean values for each sleep outcome for various values of TV+DVD screen time ( $\mathrm{min} /$ day), with TV+DVD screen time being the average over the entire 1 -year study period (ie, between-infant effects). Data are from 558 dyads with data collected during at least one postpartum follow-up; not all dyads have complete data at each timepoint. The following are the number of infants per each range of average daily TV+DVD time over the study period: $0 \mathrm{~min} /$ day: $n=124 ; 1-15 \mathrm{~min} /$ day: $n=82 ; 16-30 \mathrm{~min} /$ day: $n=52 ; 31-45 \mathrm{~min} /$ day: $\mathrm{n}=47 ; 46-60 \mathrm{~min} /$ day: $\mathrm{n}=50 ; 61-75 \mathrm{~min} /$ day: $\mathrm{n}=26 ; 76-90 \mathrm{~min} /$ day: $\mathrm{n}=24 ; 91-105 \mathrm{~min} /$ day: $\mathrm{n}=22 ; 106-120 \mathrm{~min} /$ day: $\mathrm{n}=12 ; 120+$ $\min /$ day: $n=119$.

address if screen time more (vs less) proximal to bedtime accounted for our findings.

This study included high racial and socioeconomic diversity, multiple measures of infant screen time and sleep during the first year, five screen-based activities common among infants, and a rich set of measures to adjust for potential confounding. Our study is not without limitations, however. Infant screen time and sleep were parentreported and are subject to error, the effect of which may be most pronounced for night-time awakenings. ${ }^{41}$ We did not collect data on the length of night-time awakenings and thus could not adjust night-time and 24-hour sleep for awakenings. Our measurements of infant screen time lacked the granularity needed to address timing of screen use (eg, before bedtime) and effects on sleep; parents did not report when during the day or night their infants used screens. However, our measure of infant screen time is consistent with previous studies on infant screen time and sleep (ie, usual hours per day). ${ }^{13-18}$ We also did not assess potentially different effects of different types of content, although children may not comprehend screen media content until 2 years of age. ${ }^{26}$ It is possible that unmeasured characteristics, such as child temperament, ${ }^{4243}$ may confound our reported associations. Per the methods of Curran and Bauer, ${ }^{27}$ we used simple linear regression to compute each infant's expected value of screen time at each timepoint, which does not account for the variability of those estimates in the later mixed-effects regression models. Data on both infant screen time and sleep were not available at each timepoint for each dyad; while mixed-effects regression accounts for partial missing data, our effects may be biased if the missing data process was not missing at random.

\section{CONCLUSIONS}

In this diverse, regional cohort, most infants engaged with screens from 3 to 12 months of age and infant screen time increased with age. Given that screen use behaviours track from infancy into early childhood, ${ }^{44}$ the findings highlight the need for clear messaging and intervention efforts to limit screen use and exposure during infancy. Furthermore, we found that greater amounts of infant screen time were associated with shorter night-time sleep duration when considering the between-infant effect, yet we report a non-significant within-infant effect. Studies 
to specifically assess potential causality, ideally with more intensive objective measures of infant sleep and screen time, are needed to help inform evidence-based guidelines. Continued studies are needed to understand how screen use at such a young age may affect sleep and other areas of growth and development, and our findings support the current guidelines of no screen time for infants under 18 months of age to support healthy sleep behaviours in infancy given the potential detrimental effects of screen time on infant health.

\section{Author affiliations}

${ }^{1}$ Department of Biomedical Data Science, Geisel School of Medicine, Dartmouth College, Hanover, New Hampshire, USA

${ }^{2}$ Department of Pediatrics, Geisel School of Medicine, Dartmouth College, Hanover, New Hampshire, USA

${ }^{3}$ The Dartmouth Institute, Geisel School of Medicine, Dartmouth College, Hanover, New Hampshire, USA

${ }^{4}$ Department of Public Health Sciences, Medical University of South Carolina, Charleston, South Carolina, USA

${ }^{5}$ Division of Pediatric Pulmonary and Sleep Medicine, Duke University Medical Center, Durham, North Carolina, USA

${ }^{6}$ Department of Family Medicine and Community Health, Duke University, Durham, North Carolina, USA

${ }^{7}$ Department of Health, Behavior and Society, Johns Hopkins Bloomberg School of Public Health, Baltimore, Maryland, USA

\section{Acknowledgements We thank the women and infants enrolled in the Nurture} study for their participation.

Contributors JAE and SEB-N had full access to all the data in the study and take responsibility for the integrity of the data and the accuracy of the data analysis. TO, RK and SEB-N designed the initial cohort study, obtained funding and acquired the data. JAE, AJO and SEB-N conceptualised and designed the current secondary analysis. JAE analysed the data and AJO, BN and SEB-N provided critical input during data analysis. JAE drafted the manuscript and all authors provided critical revision to the manuscript for important intellectual content. All authors read and approved the final version of the manuscript.

Funding This study was supported by a grant from the National Institutes of Health (R01DK094841) and a mentored research scientist training award from the National Institutes of Health (5K01DK117971). The sponsors had no role in the design and conduct of the study; collection, management, analysis and interpretation of the data; preparation, review or approval of the manuscript; or decision to submit the manuscript for publication.

Competing interests None declared.

Patient consent for publication Not required.

Ethics approval Ethical approval for this study was received from the Institutional Review Board of Duke University Medical Center.

Provenance and peer review Not commissioned; externally peer reviewed.

Data availability statement Data are available upon reasonable request.

Supplemental material This content has been supplied by the author(s). It has not been vetted by BMJ Publishing Group Limited (BMJ) and may not have been peer-reviewed. Any opinions or recommendations discussed are solely those of the author(s) and are not endorsed by BMJ. BMJ disclaims all liability and responsibility arising from any reliance placed on the content. Where the content includes any translated material, BMJ does not warrant the accuracy and reliability of the translations (including but not limited to local regulations, clinical guidelines, terminology, drug names and drug dosages), and is not responsible for any error and/or omissions arising from translation and adaptation or otherwise.

Open access This is an open access article distributed in accordance with the Creative Commons Attribution Non Commercial (CC BY-NC 4.0) license, which permits others to distribute, remix, adapt, build upon this work non-commercially, and license their derivative works on different terms, provided the original work is properly cited, appropriate credit is given, any changes made indicated, and the use is non-commercial. See: http://creativecommons.org/licenses/by-nc/4.0/.
ORCID iDs

Jennifer A Emond http://orcid.org/0000-0001-8684-7330

Sara E Benjamin-Neelon http://orcid.org/0000-0003-4643-2397

\section{REFERENCES}

1 Scher A. Infant sleep at 10 months of age as a window to cognitive development. Early Hum Dev 2005;81:289-92.

2 Gibson R, Elder D, Gander P. Actigraphic sleep and developmental progress of one-year-old infants. Sleep Biol Rhythms 2012;10:77-83.

3 Field T. Infant sleep problems and interventions: a review. Infant Behav Dev 2017;47:40-53.

4 Taveras EM, Rifas-Shiman SL, Oken E, et al. Short sleep duration in infancy and risk of childhood overweight. Arch Pediatr Adolesc Med 2008;162:305-11.

5 Tikotzky L, DE Marcas G, Har-Toov J, et al. Sleep and physical growth in infants during the first 6 months. J Sleep Res 2010;19:103-10.

6 Halal CSE, Matijasevich A, Howe LD, et al. Short sleep duration in the first years of life and Obesity/Overweight at age 4 years: a birth cohort study. J Pediatr 2016;168:99-103.

7 Davis KF, Parker KP, Montgomery GL. Sleep in infants and young children: Part one: normal sleep. J Pediatr Health Care 2004;18:65-71.

8 Henderson JMT, France KG, Blampied NM. The consolidation of infants' nocturnal sleep across the first year of life. Sleep Med Rev 2011;15:211-20.

9 Galland BC, Taylor BJ, Elder DE, et al. Normal sleep patterns in infants and children: a systematic review of observational studies. Sleep Med Rev 2012;16:213-22.

10 Tham EK, Schneider N, Broekman BF. Infant sleep and its relation with cognition and growth: a narrative review. Nat Sci Sleep 2017:9:135-49.

11 Mindell JA, Leichman ES, Composto J, et al. Development of infant and toddler sleep patterns: real-world data from a mobile application. J Sleep Res 2016;25:508-16.

12 Paruthi S, Brooks LJ, D'Ambrosio C, et al. Recommended amount of sleep for pediatric populations: a consensus statement of the American Academy of sleep medicine. J Clin Sleep Med 2016;12:785-6.

13 Cheung CHM, Bedford R, Saez De Urabain IR, et al. Daily touchscreen use in infants and toddlers is associated with reduced sleep and delayed sleep onset. Sci Rep 2017;7:1-7.

14 Chen B, van Dam RM, Tan CS, et al. Screen viewing behavior and sleep duration among children aged 2 and below. BMC Public Health 2019;19:59.

15 Cespedes EM, Gillman MW, Kleinman K, et al. Television viewing, bedroom television, and sleep duration from infancy to midchildhood. Pediatrics 2014;133:e1163-71.

16 Nevarez MD, Rifas-Shiman SL, Kleinman KP, et al. Associations of early life risk factors with infant sleep duration. Acad Pediatr 2010;10:187-93.

17 Przybylski AK. Digital screen time and pediatric sleep: evidence from a Preregistered cohort study. J Pediatr 2019;205:218-23.

18 Ribner AD, McHarg GG, NewFAMS Study Team. Why won't she sleep? Screen exposure and sleep patterns in young infants. Infant Behav Dev 2019;57:101334.

19 Hale L, Guan S. Screen time and sleep among school-aged children and adolescents: a systematic literature review. Sleep Med Rev 2015;21:50-8.

20 Carter B, Rees P, Hale L, et al. Association between portable screen-based media device access or use and sleep outcomes: a systematic review and meta-analysis. JAMA Pediatr 2016;170:1202-8.

21 Common Sense Media. The common sense census: media use by kids age zero to eight. Common Sense Media 2017.

22 AAP. American Academy of Pediatrics Announces New Recommendations for Children's Media Use. Am Acad Pediatr website, 2016. Available: https://www.healthychildren.org/English/ news/Pages/AAP-Announces-New-Recommendations-for-ChildrensMedia-Use.aspx

23 Reid Chassiakos YL, Radesky J, Christakis D, et al. Children and adolescents and digital media. Pediatrics 2016;138:e20162593.

24 Hipp D, Gerhardstein P, Zimmermann L. The dimensional divide: learning from TV and touchscreens during early childhood. In: Media exposure during infancy and early childhood. The effects of content and context on learning and development, 2016.

25 COUNCIL ON COMMUNICATIONS AND MEDIA. Media and young minds. Pediatrics 2016;138:e20162591. 
26 Anderson DR, Subrahmanyam K, Cognitive Impacts of Digital Media Workgroup. Digital screen media and cognitive development. Pediatrics 2017;140:S57-61.

27 Curran PJ, Bauer DJ. The disaggregation of within-person and between-person effects in longitudinal models of change. Annu Rev Psychol 2011;62:583-619.

28 Benjamin Neelon SE, Østbye T, Bennett GG, et al. Cohort profile for the nurture observational study examining associations of multiple caregivers on infant growth in the southeastern USA. BMJ Open 2017; 7:e013939.

29 Cox JL, Holden JM, Sagovsky R. Detection of postnatal depression. development of the 10-item Edinburgh postnatal depression scale. Br J Psychiatry 1987;150:782-6.

30 Coll CdeVN, Domingues MR, Stein A, et al. Efficacy of regular exercise during pregnancy on the prevention of postpartum depression: the PAMELA randomized clinical trial. JAMA Netw Open 2019;2:e186861.

31 Matheny AP, Wachs TD, Ludwig JL, et al. Bringing order out of chaos: psychometric characteristics of the confusion, hubbub, and order scale. J Appl Dev Psychol 1995;16:429-44.

32 Figueiredo B, Dias CC, Pinto TM, et al. Exclusive breastfeeding at three months and infant sleep-wake behaviors at two weeks, three and six months. Infant Behav Dev 2017:49:62-9.

$33 \mathrm{Chen} \mathrm{J}-\mathrm{H}$. Complex childcare experiences and sleep outcomes in young children. Sleep Health 2017;3:373-8.

34 Komada Y, Asaoka S, Abe T, et al. Relationship between napping pattern and nocturnal sleep among Japanese nursery school children. Sleep Med 2012;13:107-10.
35 Coyne SM, Holmgren HG, Keenan-Kroff SL, et al. Prenatal predictors of media use during infancy. Cyberpsychol Behav Soc Netw 2020;23:377-83.

36 Mersky JP, Lee CP, Gilbert RM, et al. Prevalence and correlates of maternal and infant sleep problems in a low-income US sample. Matern Child Health J 2020;24:196-203.

37 Dias CC, Figueiredo B. Unidirectional and bidirectional links between maternal depression symptoms and infant sleep problems. J Sleep Res 2021:e13363.

38 Covington LB, Patterson F, Hale LE, et al. The contributory role of the family context in early childhood sleep health: a systematic review. Sleep Health 2021;7:254-265.

39 Garrison MM, Christakis DA. The impact of a healthy media use intervention on sleep in preschool children. Pediatrics 2012;130:492-9.

40 Garrison MM, Liekweg K, Christakis DA. Media use and child sleep: the impact of content, timing, and environment. Pediatrics 2011;128:29-35.

41 Sadeh A. Commentary: comparing actigraphy and parental report as measures of children's sleep. J Pediatr Psychol 2008;33:406-7.

42 Sadeh A, Lavie P, Scher A. Sleep and temperament: maternal perceptions of temperament of Sleep-Disturbed toddlers. Early Education \& Development 1994;5:311-22.

43 Radesky JS, Silverstein M, Zuckerman B, et al. Infant self-regulation and early childhood media exposure. Pediatrics 2014;133:e1172-8.

44 Trinh M-H, Sundaram R, Robinson SL, et al. Association of Trajectory and Covariates of Children's Screen Media Time. JAMA Pediatr 2020;174:71-8. 